\title{
FORMULACIÓN DE UNA COOPERATIVA DE SERVICIOS TURÍSTICOS EN LOS MUNICIPIOS DE SOPÓ Y LA CALERA, CUNDINAMARCA (COLOMBIA)
}

\section{A COOPERATIVE TOURISM SERVICES ENTERPRISE PROJECT FOR SOPO AND LA CALERA MUNICIPALITIES, CUNDINAMARCA (COLOMBIA)}

\author{
Doris Emilia Guerrero ${ }^{1}$ \\ William Ricardo Zambrano² \\ Maria Concepción Salazar Alonso ${ }^{3}$ \\ Alvaro Lamprea Delgado ${ }^{4}$ \\ Jairo Alberto Vásquez Bernal ${ }^{5}$ \\ Jorge Sandoval Torres ${ }^{6}$ \\ Adriana Posada Arrubla ${ }^{7}$
}

\section{RESUMEN}

Propone un modelo legal y administrativo para la conformación de una cooperativa de trabajo asociado de un corredor turístico, en los municipios de Sopó y La Calera (Cundinamarca-Colombia). Se tuvo en cuenta

${ }^{1}$ Abogada. Filósofa Docente Investigador. Ingeniería Comercial U.D.C.A. Email: dorisgue@udca.edu.co

2 Comunicador Social - Periodista, M.Sc Comunicación, Docente Investigador Ingeniería Comercial. U.D.C.A. Email: zambrano william@hotmail.com

${ }^{3}$ Economista - Bibliotecóloga- Docente Investigador Ingeniería Comercial. U.D.C.A. Email: masalazar@udca.edu.co

${ }^{4}$ Profesional Negocios Internacionales. Docente Investigador. Ingeniería Comercial U.D.C.A. Email: alamprea@udca.edu.co

Mercadotecnista, Esp. Gestión Social y Ambiental, Docente Investigador Ingeniería Comercial U.D.C.A. Email: jvasquez@udca.edu.co

- Economista-Mercadotecnista, M.Sc. Docencia Universitaria. Docente Investigador Ingeniería Comercial. U.D.C.A. Email: jorhumsan@hotmail.com

${ }^{7}$ Economista Agrícola, M.Sc. Planeación Urbana y Regional, decana Facultad Ingeniería Comercial. U.D.C.A. Email: aposada@udca.edu.co las necesidades sociales, las características naturales y culturales de la región, la oportunidad para sumar esfuerzos intermunicipales, el apoyo de la empresa pública y privada, con el ánimo de organizar competitivamente el ofrecimiento de sus servicios turísticos y ecoturísticos. Pretende integrar no sólo a los futuros asociados, sino a los procesos de fusión subregional para maximizar beneficios sociales, económicos y ambientales a bajo costo. Para cumplir con este propósito, se realizó una investigación exploratoria, lo que facilitó el aporte de información para la descripción, análisis, interpretación y argumentación del objeto de estudio, el cual deja como conclusión, que la constitución de una empresa de economía solidaria es un modelo viable de asociación, que hace posible articular la necesidad de la comunidad, como estrategia para su desarrollo.

Palabras clave: Precooperativa, cooperativa, asociación, turismo, ecoturismo.

\section{SUMMARY}

To create an associative cooperative enterprise for the tourist corridor of the municipalities of Sopo and $\mathrm{La}$ Calera (Cundinamarca-Colombia), a legal and administrative model is proposed. For this purpose, the social necessities, the natural and cultural characteristics were 
taken into account, as well as the opportunity to sum up municipalities efforts, to the support of the private sector, in order to organize competitively the offer of tourism and ecotourism services. It was attempted not only to integrate the future confederates, but also merge them with the sub-regional fusion processes in order to maximize social, economic and environmental benefits at low cost. To achieve this target an exploratory research was conducted, gathering the data required for the description, analysis, assessment and subject discussion, leading to the conclusion that the establishment of a solidary economy enterprise is a feasible association project that would facilitate the fulfillment of the people needs as a development strategy.

Key words: Pre-cooperative, cooperative, association, tourism, ecotourism.

\section{INTRODUCCIÓN}

El crecimiento turístico en el mundo, se enfrenta a un gran reto para convertirse en un sector económico sostenible que sirva para proteger la riqueza natural del planeta y mejorar las condiciones de vida de las poblaciones más desfavorecidas. Por esta razón, "las organizaciones internacionales han mostrado interés en impulsar el turismo sostenible que no afecte el ambiente y los recursos naturales" (Betancourt, 2004).

Esta actividad, se sustenta en los principios de estabilidad en el desarrollo local y global en su permanente evolución en el largo plazo, en la equidad, en la distribución de sus beneficios, además "en la previsión y control eficaz de los impactos no deseables sobre la economía, la cultura, el medio ambiente natural y social del receptor" (Ayala, 2006), sin dejar de atraer y de beneficiar a los turistas de los distintos países emisores.

Se alerta sobre la necesidad de proteger las reservas, la planificación y la participación de las comunidades nativas, sobre todo en aquellas regiones de alta biodiversidad, donde la riqueza ecológica convive con la pobreza humana, de acuerdo con el Informe de la cumbre mundial de desarrollo sostenible, celebrada en Johannesburgo-Sudáfrica (ONU, 2003).

El turismo local debe proponer una oferta competitiva que, al mismo tiempo, satisfaga las necesidades de los turistas y contribuya al progreso y bienestar de sus habitantes (Morales, 2006). Para lograr el desarrollo económico y local es necesario proponer, según la Comisión Europea (2007), una gestión integrada de la calidad, ofreciendo una experiencia única y original al visitante, satisfaciendo las legítimas aspiraciones de la población a un desarrollo económico, social, armonioso y respetuoso con el ambiente.

Por lo anterior, se requiere aunar esfuerzos en el manejo y en la administración adecuados de los recursos ambientales, físicos, humanos y financieros, mediante la creación de organizaciones que los administren, eficientemente, en beneficio de la comunidad. Desde lo institucional, deben concurrir actores de los sectores públicos y privados encargados de funciones, como planificar, certificar, acreditar y fomentar la actividad del sector, en últimas, "garantizar y/o proporcionar el acceso, calidad de la infraestructura, bienes, servicios utilizados y ofrecidos a los visitantes" (Muñoz, 2004).

La Organización Mundial del Turismo (OMT, 2000) establece que, con el fin de asegurar las actividades turísticas, se requiere incentivar las oportunidades de empleo estable y garantizar la obtención de ingresos y servicios sociales para las comunidades, estrechando la colaboración entre los diferentes sectores, a través de modelos de cooperación.

Ballén (2001) afirma que "esta actividad se ha convertido en sinónimo de crecimiento y fuente de ingresos para las naciones en vías de desarrollo como Colombia”. Así, las diferentes regiones de nuestro país, entre ellas la conformada por los municipios de La Calera y Sopó, en Cundinamarca, privilegian en sus planes de desarrollo a la actividad turística (PDES, 2004-2007) y (PDES, 20042008); sin embargo, no contemplan el establecimiento de alianzas estratégicas ni la conformación de empresas administradoras para la prestación de estos servicios.

A partir de esta carencia, se propone la factibilidad de una oferta competitiva, a través de la creación de una cooperativa de trabajo asociado. Para hacerla viable, los prestadores de servicios turísticos de Sopó y La Calera, se deben desprender del tradicional rebusque e informalidad en la prestación del servicio para el aprovechamiento de las ventajas competitivas de su patrimonio y se preparen hacia "una forma empresarial especializada que cohesione sus prácticas, conocimientos, recursos" (Bejarano, 2004). 
De los anteriores propósitos, se originaron las siguientes preguntas orientadoras para la formulación de una cooperativa de trabajo asociado, como estrategia competitiva de servicios turísticos: cel turismo se puede convertir en un sector económico sostenible en los municipios de Sopó y La Calera?, ćse necesita unir esfuerzos para el manejo y la administración adecuado de los recursos de los municipios, con el fin de proponer la creación de organizaciones que los administren en beneficio de la comunidad?, cestán los oferentes turísticos de estos municipios preparados para conformar una unidad empresarial organizada y especializada que cohesione sus prácticas y conocimientos?, ces viable la creación de una cooperativa de trabajo asociado, con el fin de proporcionar una oferta competitiva de los servicios de la región?, ces necesario la constitución de una pre-cooperativa que permita el tránsito hacia una cooperativa para la administración de un corredor turístico de Sopó y La Calera?

\section{MATERIALES Y MÉTODOS}

Se realizó una investigación interdisciplinar de tipo exploratorio de carácter cualitativo y cuantitativo, que permitió la descripción, el análisis, la interpretación y argumentación de la información obtenida, sobre la variable dependiente de la creación de una cooperativa de trabajo asociado, que posibilite interrelacionarla con la situación, necesidades y desarrollos municipales.

La Calera situada al oriente del departamento de Cundinamarca y al noreste de Bogotá-Colombia, su centro urbano está a los $4^{\circ} 43^{\prime} 17^{\prime \prime}$ de latitud norte y $73^{\circ} 58^{\prime}$ de longitud oeste de Greenwich; en arco $0^{\circ} 06^{\prime} 29^{\prime \prime}$ este. Limita por el norte con los Municipios de Guasca, Sopó y Chía; el oriente con Guasca; al occidente con Bogotá y al sur con el municipio de Choachí y Bogotá. Su extensión es de $485 \mathrm{~km}^{2}$, según datos suministrados por el Departamento Administrativo Nacional de Estadística (DANE, 2007), con altitud $2718 \mathrm{msnm}, 14^{\circ} \mathrm{C}, 24.841$ habitantes.

Sopó ubicado en el departamento de Cundinamarca a $39 \mathrm{~km}$ al noreste de Bogotá capital de Colombia, se ubica entre los $4^{\circ} 5450^{\circ}$ de latitud norte y a los $73^{\circ} 5706^{`}$ de longitud oeste. Limita por el norte con Tocancipá, al oriente con Guasca, al sur con La Calera y al occidente con Cajicá y Chía; extensión: 111,5km2, con altitud 2.650msnm; $13^{\circ} \mathrm{C} ; 21.906$ habitantes, según el DANE (2007).
La información, se recolectó de la realidad, permitiendo desarrollar un método abierto, aplicando encuestas y entrevistas en profundidad, lo que facilitó el aporte de información "en función del contexto y para fundamentar el estudio" (Tamayo E Tamayo, 2002). Para la determinación de la población, se seleccionaron dos grupos, como tamaño de la muestra, para la aplicación de la encuesta y de la entrevista. El primero, integrado por los propietarios y/o administradores de los establecimientos de comercio de los municipios referenciados, quienes participarán de los beneficios que reporte el desarrollo turístico. Para tal fin, se acudió a la recopilación de las empresas registradas en el año 2006, suministrada por la Secretaría de Desarrollo Económico y Turístico y por Planeación Municipal, arrojando los siguientes datos para unidades comerciales: 390 en Sopó y 167 en La Calera, para un total de 557.

A partir de estos consolidados, se tomó una muestra, que corresponde a un subconjunto de los miembros de una población. La información, se extrajo de dicho subconjunto y se empleó para estimar diversas características de la población total, "lo ideal es que la muestra de la población de la cual se obtiene información sea un corte transversal representativo del total de unidades objeto de estudio" (McDaniel, 2001).

El proceso de muestreo implementado para la selección de la población en cuestión fue el estratificado proporcional, dividiéndola en grupos homogéneos de unidades de negocio y de turistas. Las variables específicas para unidades de negocio fueron: identificación de atractivos turísticos, prestación de servicios, intención para conformar una empresa administradora y participación en capacitación, servicios turísticos que brinda, frecuencia, costo en la prestación y número de personas atendidas. En cuanto a las variables identificadas para el grupo de turistas fueron: participación en actividades turísticas en los municipios, conocimiento de servicios y atractivos, lugares y frecuencia utilizados para su práctica, intención de conocer el corredor turístico entre La Calera y Sopó.

Resultado de los datos anteriores, se escogió en forma aleatoria un total de 125 unidades, objeto de estudio. Para tal efecto, se formularon las siguientes preguntas a través de encuesta: ¿su empresa o establecimiento ofrece servicios turísticos?, ¿participaría en un proyecto para la conformación de una empresa administradora de un corredor turístico en La Calera y Sopó? y cंparticiparía en un proceso de capacitación y asesoría empresarial? 
De otra parte, mediante entrevista, se formularon los siguientes cuestionamientos: ¿cuáles son los factores de éxito para la prestación del servicio?, ¿cuáles servicios turísticos brinda?, cंqué días los presta?, ¿cuál es el número de personas promedio que trabajan en el establecimiento?, ¿cuántas atiende diariamente?, ¿cuál es el costo de los servicios ofrecidos?

En cuanto a los turistas, se determinó que la población objeto de estudio es infinita, por tal razón, se seleccionó de manera aleatoria, soportada en las estadísticas suministradas por la Secretaría de Desarrollo Económico y Turístico y de Planeación Municipal (2006-2007); en cuanto a número de turistas visitantes. El tamaño de la muestra correspondió a los siguientes individuos encuestados: 105, en Sopó y 115, en La Calera, para un total de 220, a los cuales se les formuló preguntas cerradas y entrevistas abiertas a turistas: ¿participa en actividades turísticas en Sopó y La Calera?, ċrecorrería un corredor turístico entre estos dos municipios? y cंconoce las actividades turísticas que se realizan allí?

En cuanto a la entrevista, se aplicaron las siguientes: ¿qué tipo de turismo practica en estos municipios?, ¿cuáles son los lugares preferidos por usted para hacer turismo en Sopó y La Calera?, ċcon qué frecuencia los visita?, ¿̇uáles son las razones para visitarlos?, ¿qué servicios turísticos utiliza? y ¿qué atractivos turísticos conoce de estos municipios?

Los parámetros que guiaron el método de integración de la información tanto cuantitativa como cualitativa fueron los siguientes: selección de niveles de análisis para identificar la capacidad en la prestación del servicio turístico, necesidades de recreación de los usuarios; observación para la recolección de información para identificar categorías homogéneas y, por último, la realización de un proceso de depuración de la información, para la organización, la planeación, la evaluación y la interpretación de datos fundamentales que guiaron el estudio.

\section{RESULTADOS Y DISCUSIÓN}

Según las tablas 1 y 2 , la tendencia a participar en una empresa administradora de un corredor turístico entre los municipios de la Calera y Sopó es mayoritaria. Las razones que se expresaron para la elección de este tipo de asociación fueron: el potencial de los municipios de acuerdo con sus atractivos, el conocimiento del servicio, la experiencia en el ramo y la unión de fuerzas para enfrentarse competitivamente.

De otra parte, se tuvo en cuenta dos variables para la elección del marco legal y administrativo: de un lado, la encuesta y la entrevista, por otro, el análisis e interpretación de las leyes apropiadas. Sobre el marco legal, se diseñaron dos escenarios probables que permitiera sopesar las ventajas y las desventajas de los tipos de asociación posible: de economía capitalista y sin ánimo de lucro. Posteriormente, estos fueron socializados con los interesados oferentes encuestados y entrevistados, dejando como conclusión su interés por la conformación de una asociación de economía solidaria del tipo cooperativo de trabajo asociado (tabla 3).

Lo anterior, permitió tener en cuenta las necesidades sociales, las características naturales y culturales de la región, la oportunidad para sumar esfuerzos intermunicipales y el apoyo de la empresa pública y privada. De igual manera, se analizaron otros factores como, la riqueza de la biodiversidad y la forma cómo las comunidades interactúan en su entorno.

Adicionalmente, se recurrió al análisis legal como se mencionó, lo que generó la interpretación de la Ley 300 de 1996, denominada General de Turismo, por medio de la cual se define la actividad turística como una industria esencial para el desarrollo del país, en especial de las diferentes entidades territoriales y regionales, cumpliendo así con su función social.

El estudio enfatizó en la importancia de considerar la participación de las empresas entre los diferentes tipos de agremiación solidaria, para organizar y administrar los recursos del corredor turístico de los dos municipios, con el fin de generar desarrollo socio-económico en la comunidad y no enfocado exclusivamente al sector turístico tradicional. Según Aguirre (1997), "estar organizada como cooperativa de trabajo asociado permite comprender su objeto social tendiente a satisfacer necesidades de sus asociados" y el desarrollo de obras de servicio comunitario.

En este orden de ideas, el Departamento Administrativo Nacional de Cooperativas (DANCOOP, 1997) afirma que establecer un vínculo asociativo fundado en los 
Tabla 1. Entrevista a oferentes y a visitantes de servicios turísticos en Sopó y La Calera.

\begin{tabular}{|c|c|c|c|}
\hline PREGUNTAS A OFERENTES & Preferencia & Tendencia & $\%$ \\
\hline \multirow{2}{*}{ ¿Cuáles son los factores de éxito para la prestación del servicio? } & La atención al público & 75 & 35,05 \\
\hline & Calidad & 38 & 17,76 \\
\hline \multirow{2}{*}{ ¿Cuáles servicios turísticos brinda? } & Gastronomía & 26 & 23,2 \\
\hline & Venta artesanías & 3 & 2,7 \\
\hline \multirow{2}{*}{ ¿Qué días los presta? } & Todos los días & 105 & 84 \\
\hline & Fines de semana & 14 & 11,2 \\
\hline \multirow{2}{*}{$\begin{array}{l}\text { ¿Cuál es el número de personas promedio que trabajan en el } \\
\text { establecimiento? }\end{array}$} & $1 \mathrm{a} 4$ & 100 & 79,36 \\
\hline & 5 a 8 & 19 & 15,07 \\
\hline \multirow{2}{*}{ ¿Cuántas atiende diariamente? } & $1 \mathrm{a} 4$ & 100 & 79,36 \\
\hline & 5 a 8 & 19 & 15,07 \\
\hline \multirow{2}{*}{ ¿Cuál es el costo de los servicios ofrecidos? } & $500-24.714$ & 70 & 56 \\
\hline & 24.715- 48.928 & 26 & 20,8 \\
\hline \multicolumn{4}{|l|}{ PREGUNTA A TURÍSTAS } \\
\hline \multirow{2}{*}{ ¿Qué tipo de turismo practica en estos municipios? } & Ecológico & 81 & 35,1 \\
\hline & De aventura & 36 & 15,6 \\
\hline \multirow{2}{*}{$\begin{array}{l}\text { ¿Cuáles son los lugares preferidos por usted para hacer turismo en } \\
\text { Sopó y La Calera? }\end{array}$} & Pionono & 44 & 20 \\
\hline & Religiosos & 42 & 19 \\
\hline \multirow{2}{*}{ ¿Con qué frecuencia los visita? } & Cada mes & 77 & 35 \\
\hline & Cada 15 días & 22 & 10 \\
\hline \multirow{2}{*}{ ¿Cuáles son las razones para visitarlos? } & Conocer & 40 & 18,1 \\
\hline & Religioso & 35 & 15,9 \\
\hline \multirow{2}{*}{ ¿Qué servicios turísticos utiliza? } & Gastronómicos & 90 & 40,9 \\
\hline & Artesanales & 41 & 18,6 \\
\hline \multirow{2}{*}{ ¿Qué atractivos turísticos conoce de estos municipios? } & Pionono & 60 & 27,2 \\
\hline & Alpina & 52 & 23,6 \\
\hline
\end{tabular}

Tabla 2. Encuesta a oferentes y a visitantes a los municipios de Sopó y La Calera.

\begin{tabular}{|l|c|c|c|c|}
\hline \multicolumn{1}{|c|}{ PREGUNTA A TURISTAS } & SI & $\%$ & NO & $\%$ \\
\hline ¿Participa en actividades turísticas en Sopó y La Calera? & 187 & 85 & 33 & 15 \\
\hline ¿Recorrería un corredor turístico entre estos dos municipios? & 199 & 90.4 & 21 & 9.6 \\
\hline ¿Conoce las actividades turísticas que se realizan allí? & 154 & 70 & 66 & 30 \\
\hline & SIEGUNTA A OFERENTES & $\%$ & NO & $\%$ \\
\hline ¿Su empresa o establecimiento ofrece servicios turísticos? & 119 & 95,2 & 6 & 4,8 \\
\hline $\begin{array}{l}\text { ¿Participaría en un proyecto para la conformación de una empresa } \\
\text { administradora de un corredor turístico en La Calera y Sopó? }\end{array}$ & 120 & 96 & 5 & 4 \\
\hline ¿Participación en un proceso de capacitación y asesoría empresarial? & 115 & 92 & 10 & 8 \\
\hline
\end{tabular}


Tabla 3. Escenarios de asociación para la administración del corredor turístico de Sopó y La Calera.

\begin{tabular}{|c|c|}
\hline $\begin{array}{l}\text { Escenario } 1 . \\
\text { Empresa de Economía Capitalista }\end{array}$ & $\begin{array}{l}\text { Escenario } 2 . \\
\text { Empresa de Economía Solidaria }\end{array}$ \\
\hline 1. Su principal objetivo es la ganancia & 1. Su principal objetivo es la persona y la comunidad. \\
\hline $\begin{array}{l}\text { 2. En la asamblea de socios, cada accionista o aportante tiene derecho } \\
\text { a tantos votos como acciones tenga }\end{array}$ & $\begin{array}{l}\text { 2. En la Asamblea un asociado sólo se tiene derecho a un voto sin } \\
\text { importar el capital que se tenga. }\end{array}$ \\
\hline 3. Los trabajadores son asalariados solamente & $\begin{array}{l}\text { 3. Los trabajadores son los mismos asociados, aportantes } 0 \\
\text { propietarios. }\end{array}$ \\
\hline $\begin{array}{l}\text { 4. Las ganancias son repartidas según los aportes o acciones que cada } \\
\text { socio tengan sin importar que unos trabajen más que otros. }\end{array}$ & $\begin{array}{l}\text { 4. Los excedentes son: en primer lugar, para prestar servicios y } \\
\text { segundo, para distribuirlos entre sus asociados. }\end{array}$ \\
\hline 5. El trabajo es asalariado & 5. El trabajo es autogestionario \\
\hline 6. Prima el capital sobre el trabajo & 6. Prima el trabajo sobre el capital \\
\hline 7. Los trabajadores son asalariados generalmente & 7. Los asociados trabajadores son los mismos dueños \\
\hline 8. El socio que tenga más acciones tiene más poder de decisión & 8. Hay democracia participativa \\
\hline 9. Cada asalariado trabaja por un sueldo & 9. Se trabaja por un fin grupal \\
\hline
\end{tabular}

principios y fines del cooperativismo, debe incluir en sus estatutos de funcionamiento la ausencia de ánimo de lucro, movida por la solidaridad, el servicio social; garantizar la igualdad de derechos y obligaciones de sus miembros sin consideración a sus cuotas; establecer en sus estatutos un monto mínimo de aportes sociales no reducibles; integrarse social y económicamente sin perjuicio de sus vínculos con otras entidades.

Los anteriores son motivos para privilegiar este tipo de asociación como aquella que permite sumar fortalezas individuales para competir en el mercado de servicios turísticos en la región. Si se tiene en cuenta que las cooperativas de trabajo asociado se abren paso para ser aceptadas como integrantes de un mercado competitivo en la producción de bienes y servicios, este escenario es posible alcanzar mediante la construcción sistemática de objetivos claros, aplicación de recursos y acciones.

Lo descrito, se convierte en elementos esenciales de la estrategia de constitución, funcionamiento y consolidación de este modelo de asociación. Así mismo, es el camino a recorrer, ya que en toda empresa debe existir coherencia y consistencia interna entre los objetivos-metas, medios-recursos, acciones-programas, requerimientos necesarios para validar cualquier estrategia empresarial que ubique la cooperativa idóneamente, confiriéndole identidad, capacidad y posibilidades de éxito en el mercado.
De acuerdo con los resultados (tabla 1 y 2), la población encuestada y entrevistada, expresó su deseo de iniciar un proceso de capacitación y asesoría empresarial, que reflejará los intereses de la comunidad y la capacidad para ofrecer el servicio. Según el resultado obtenido, se consideró oportuno establecer un proceso de aprendizaje y de adaptación a formas legales de asociación de los futuros miembros de la cooperativa, para lo cual, se propuso un periodo de transición anterior al funcionamiento de la misma, de tal manera que se planteó la conformación de una precooperativa.

En el mismo sentido, se compartió con los municipios el funcionamiento inicial de estas entidades transitorias, pues ellas deben evolucionar hacia cooperativa plena en un plazo de cinco años prorrogables y la aprobación de 2/3 partes de los asociados (Comité Interinstitucional de Economía Solidaria y Empleo, 2000). Teniendo en cuenta que estas realizan actividades permitidas a las cooperativas y que por carecer de capacidad económica, educativa, administrativa o técnica, no están en posibilidad inmediata de organizarse como tales, conviene iniciar actividades con un régimen legal transitorio. Por lo anterior, se les fijan como objetivos a estas entidades: educar social y económicamente a sus asociados, organizar la prestación de servicios, suministrar bienes dentro del espíritu solidario, desarrollar procesos de formación, capacitación, adiestramiento de sus miembros y adelantar los procesos evolutivos hacia cooperativa (Bulla, 2000). 
Para que la asociación de economía solidaria formulada pueda alcanzar sus objetivos, se propone un diseño organizacional acorde con las necesidades del entorno y las exigencias legales establecidas para una cooperativa de trabajo asociado. Así, se planteó la configuración de la estructura organizacional representada en comités que componen inicialmente la precooperativa y posteriormente, la cooperativa. Su operación incluye las funciones y actividades necesarias para cumplir sus metas, su estructura básica, asignación de tareas entre comités, divisiones, equipos y cargos (Arbelaéz, 2003). En la figura 1, se presenta el diseño organizacional conforme a las exigencias legales establecidas para las cooperativas de trabajo asociado. En éste, se contempló la estructura administrativa con visión comercial, un estudio financiero y técnico integrado a los servicios turísticos, mediante procesos comunicativos.

En cuanto al aspecto mercadológico, se identificó un enfoque social enmarcado desde dos puntos de vista; el primero, la aplicación y el desarrollo de estrategias de marketing para instituciones sin ánimo de lucro; el segundo, el tema de las consecuencias de orden social del mismo, el cual hace referencia a la deseabilidad y a la honestidad de las actividades comerciales, de tal manera que lo ecológico y el consumo sean ligados e integrados desde esta perspectiva (Cobra, 2000).

Para el análisis y construcción de la estructura financiera, se utilizó el Plan Único de Cuentas (PUC, 2006) para las empresas de economía solidaria, como norma internacional y la estructura contable establecida en la ley 79 de 1988, capítulo 8, numeral 2, establecidos para las precooperativas y cooperativas de trabajo asociado, de tal manera que determine la viabilidad de los resultados de la actividad turística y la repercusión de estos en la composición del capital social.

Son primordiales para consolidar la cooperativa administradora del corredor turístico: sus objetivos,

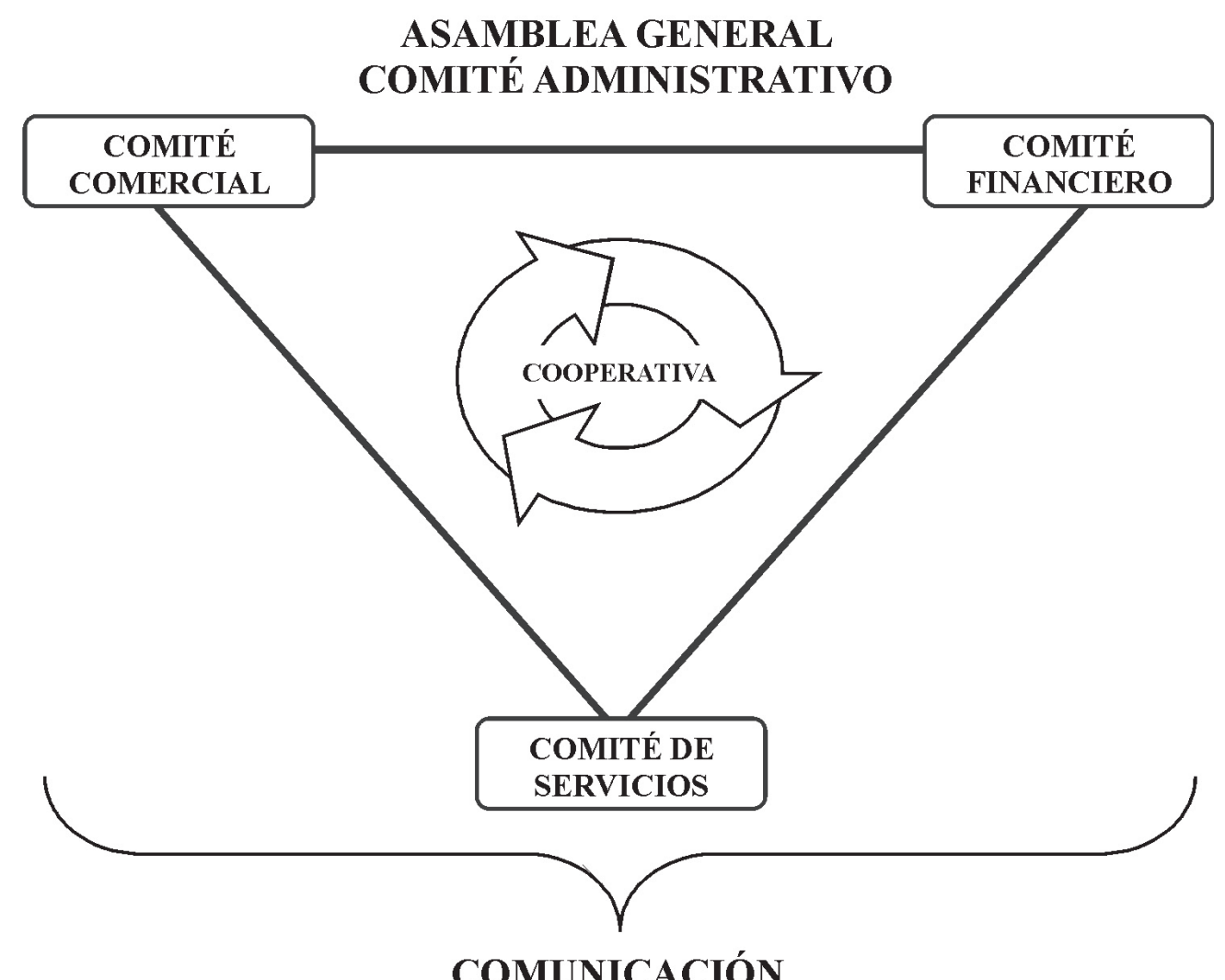

Figura 1. Diseño organizacional de la cooperativa de trabajo asociado. 
estrategias, tamaño; división del trabajo, coordinación de los comités, las personas que deben realizar la función y los estilos de comunicación e interacción, la forma de brindar el servicio turístico, los cambios que ocurran en el ambiente externo de los municipios referenciados; oportunidades, amenazas, coacciones y contingencias.

Fruto de los resultados aportados por las diferentes técnicas de recolección de información, se determinaron limitantes que podrán impedir llevar a la realidad la creación de la cooperativa de trabajo asociado para la administración del corredor turístico de Sopó y La Calera: no se identifica quién liderará el proceso de convocatoria para la iniciación de las actividades de la precooperativa; qué estrategias cambiarán el paradigma tradicional en la prestación del servicio turístico hacia la formulación de actividades mancomunadas y planeadas y qué entidad apoyará la promoción, la constitución y el desarrollo de esta nueva estrategia turística.

\section{CONCLUSIONES}

El turismo representa una buena oportunidad para contribuir al desarrollo social y económico de los municipios mencionados, pero requiere de una estrategia interdisciplinaria, participativa, dirigida a la articulación del patrimonio con la sociedad y a la preparación de ésta para mantener los recursos en forma sustentable.

Las experiencias recopiladas en el estudio mostraron que los gobiernos, la industria del turismo, los turistas, las organizaciones dedicadas al desarrollo, la sociedad civil y el sector privado deben trabajar conjuntamente con la población, para orientar el turismo hacia un rumbo más sostenible de acuerdo con modelos de asociación y cooperativismo que son de reciente implementación en las empresas ecoturísticas.

Teniendo en cuenta que los prestadores de servicios turísticos del Corredor Sopó y La Calera desempeñan papel primordial en la ejecución y administración del corredor, se propone una práctica empresarial solidaria, cuyo funcionamiento responda a intereses colectivos de su fuerza laboral y empresarial disponible para cumplir con los objetivos de impulsar el desarrollo local y nacional.
En el estudio, se estableció que las cooperativas de trabajo asociado, se presentan como una opción viable para el corredor citado. En este sentido, enfatizó que una empresa de economía solidaria, como es una cooperativa de trabajo asociado, es una persona jurídica organizada para realizar actividades sin ánimo de lucro, en las cuales los trabajadores o los usuarios, según el caso, son simultáneamente sus aportantes y sus gestores.

La estructura organizacional de la cooperativa de trabajo asociado encargada de administrar el corredor turístico Sopó y La Calera refleja su configuración estructural, sus órganos, sus relaciones de interdependencia, su funcionamiento, sus procesos de actividades y su coordinación, capaces de conducir a la consecución de los objetivos cooperativos. La estructura organizacional constituye una de las prioridades de la administración de este tipo de asociación, que sirve como base de operación, decisión y coordinación.

De la misma manera, se presentaron alternativas para la constitución de una empresa administradora, se anexó un esquema legal y administrativo a partir de la necesidad y de la realidad, proponiendo a las cooperativas de trabajo asociado como una opción posible, pues éstas, en desarrollo del acuerdo cooperativo, integran voluntariamente a sus asociados para la ejecución de labores materiales o intelectuales de conformidad con las aptitudes y capacidades de los asociados y requerimientos de los cargos (figura 2), acatando las regulaciones que establezcan los órganos de administración de éstas y sin sujeción a la legislación laboral ordinaria, puesto que las estructuras administrativas definen su gobernabilidad.

El futuro de las cooperativas de trabajo asociado es factible si se adelanta en función de las acciones planteadas, las cuales conducirán hacia el cumplimiento de estrategias, pero se debe complementar con recursos físicos, humanos, tecnológicos, económicos y financieros, con el seguimiento permanente en su proceso para que sea efectivo su cumplimiento.

A las universidades, les corresponden asumir un papel promotor de las entidades de economía solidaria, en la forma de cooperativas de trabajo asociado, como una opción valida de estructuración de un nuevo pensamiento empresarial, para todos los integrantes de 


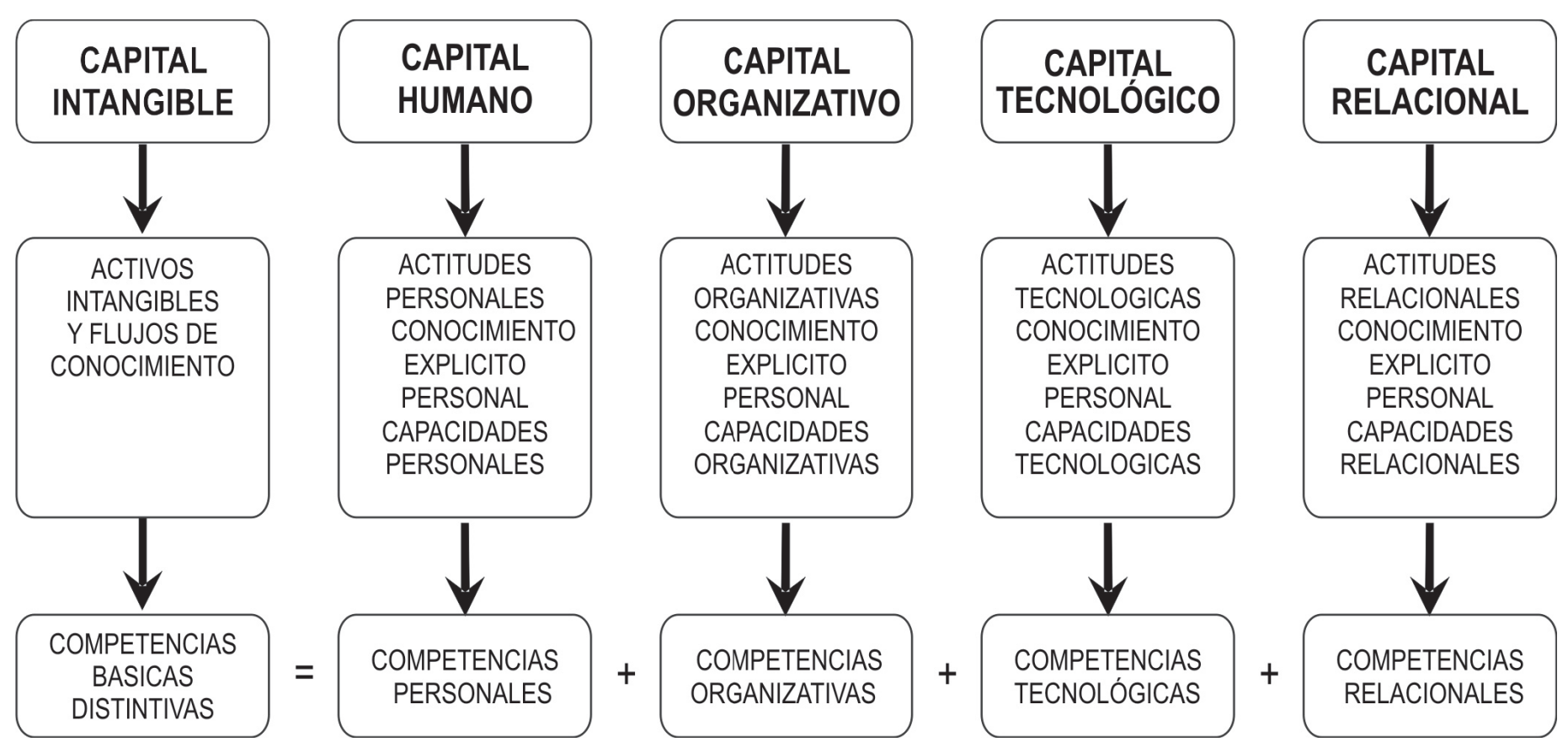

Figura 2. Requerimientos para la integración de los asociados de la precooperativa.

la comunidad donde actúa, de tal manera, que su papel en la constitución, puesta en marcha y evaluación de sus acciones sea definitiva. Igualmente, se requiere conjugar experiencias y saberes transdisciplinarios que permitan, mediante diálogos, establecer y proyectar nuevas líneas de investigación sobre este tema, vinculando a la comunidad en general.

De los resultados obtenidos surgen interrogantes que se pueden convertir en objeto de estudio de futuras investigaciones en el tema: ciestán preparadas las comunidades para entender y aprovechar de manera sostenible sus potencialidades ecoturísticas?, ces posible articular exitosamente diferentes opciones de corredores turísticos entre sitios distantes del país? y cile interesa a los Municipios identificar al turismo como opción de integrar iniciativas de cara a la internacionalización de los servicios?

\section{BIBLIOGRAFÍA}

AGUIRRE, B. 1997. La administración científica de la empresa cooperativa, como factor de desarrollo del sector cooperativo en Colombia. Bogotá: Mimeógrafo. 182p.
ARBELÁEZ, N. 2003. Conceptualización y Administración Cooperativa. Bogotá: ProUlconal.125p.

AYALA, H. 2006. Contradicciones entre turismo, economía y ecología. Rev. Turismo y Ecología. 133(2):6988. Disponible desde Internet en: http://search. ebscohost.com/login. aspx?direct $=$ trueE $\mathrm{db}=\mathrm{zbh}$ EAN $=18810155$ Eamp;lang = esEsite = ehost-live (con acceso 06/06/07).

BALLÉN, R. 2001. Aspectos fundamentales de las empresas asociativas de trabajo y cooperativas de trabajo asociado. Bogotá: Ministerio de la Protección Social, Dirección Territorial de Cundinamarca. Grupo de empleo y seguridad social. 343p.

BEJARANO, A. 2004. Cooperativismo, región y desarrollo: un planteamiento estratégico. Ibagué, Colombia: Ruecolor.134p.

BETANCOURT, M. 2004. Esquema de planificación y gestión integral del turismo desde los territorios. 3(2):1-20. Disponible desde Internet en: http://search.ebscohost.com/login.aspx?direct $=$ true $E \mathrm{db}=\mathrm{z}$ bhEAN $=18429047 E$ amp;lang = esEsite = ehostlive (con acceso 17/09/07). 
BULLA, S. 2000. Documentos de Trabajo asociado. Ministerio de la Protección Social. Bogotá. 236p.

COBRA, M. 2000. El Marketing de Servicios, las estrategias para turismo, finanzas, salud y comunicación. México: Editorial Mc Graw Hill. 370p.

COMISIÓN EUROPEA. 2007. Por un turismo de calidad. Gestión integrada de la calidad de los destinos turísticos. p.1-16. Disponible desde Internet en: ec.europa.eu/enterprise/library/lib-tourism/iqmsummary/urban_es.pdf. (con acceso 11/12/07).

COMITÉ INTERINSTITUCIONAL DE ECONOMÍA SOLIDARIA Y EMPLEO. 2000. Documentos de trabajo DANCOOP. 32p.

CONGRESO DELA REPÚBLICADE COLOMBIA. Ley 300. Diario Oficial No. 42.845, de 30 de Julio de 1996.

CONGRESO DE LA REPÚBLICA DE COLOMBIA. Ley 79. Diario Oficial No 38.648, del 10 de enero de 1989.

DEPARTAMENTO ADMINISTRATIVO NACIONAL DE COOPERATIVAS. DANCOOP 1997. Como organizar una empresa de economía solidaria, convenio con international monetary fund, Bogotá. 79p.

DEPARTAMENTO ADMINISTRATIVO NACIONAL DE ESTADÍSTICA, DANE. 2007. Disponible desde Internet en http://www.dane.gov.co/ (con acceso 08/10/07).

MCDANIEL, C. 2001. Investigación de Mercados Contemporánea. 4 ed. Bogotá: Internacional Thomson. 405p.

MORALES, H. 2006. Turismo comunitario: una nueva alternativa de desarrollo indígena. Rev. Antrop. Iberoamericana. 1(2):249-264. Disponible desde Internet en: http://www.aibr.org/antropologia/01v02/ articulos/010202.pdf. (con acceso 08/11/07).
MUÑOZ, P. 2004. Evaluación de Paisaje: una herramienta de gestión ambiental. Rev. Historia Natural. 75 (1):139-156.

ORGANIZACIÓN MUNDIAL DE TURISMO. OMT. 2000. Observaciones sobre el Turismo Internacional. El desarrollo sostenible. España 629p.

ORGANIZACIÓN DE NACIONES UINIDAS. ONU. 2003. Informe de la cumbre mundial sobre el desarrollo sostenible. Johannesburgo-Sudáfrica 2002. Publicación de la Naciones Unidas. ONU. Nueva York. 10p.

PLAN DE DESARROLLO DEL MUNICIPIO DE SOPÓ. PDES 2004-2008. Sopó unido y seguro. Disponible desde Internet en: http://www.sopo.gov.co/infoNoticia.asp?IdNot=118 (con acceso 08/06/07).

PLAN DE DESARROLLO ECONÓMICO Y SOCIAL DE LA CALERA. PDES 2004-2007. La Calera para todos. Disponible desde Internet en: http://www. sopo.gov.co/infoNoticia.asp?IdNot $=118$ (con acceso 18/05/07).

PLAN ÚNICO DE CUENTA. PUC. 2006. Disponible desde Internet en: http://www.supersolidaria.gov.co/normatividad/puc.asp (con acceso 06/06/07).

SECRETARÍA DE DESARROLLO ECONÓMICOY TURÍSTICO, Planeación Municipal. 2006-2007. Disponible desde Internet en: http://www.cundinamarca. gov.co/cundinamarca/municipios/frm_municipio. asp?codigo $=49$ (con acceso 06/08/07).

TAMAYO \& TAMAYO, M. 2002. El proceso de la Investigación Científica. 4 México: Limusa. 231p.

Recibido: Enero 15 de 2008

Aceptado: Abril 25 de 2008 